\title{
Emission modes in electro co-flow
}

\author{
Cite as: Phys. Fluids 31, 082009 (2019); doi: 10.1063/1.5121714 \\ Submitted: 26 July 2019 - Accepted: 2 August 2019 • \\ Published Online: 30 August 2019
}

J. Guerrero, ${ }^{1}$ A. J. Hijano, ${ }^{2}$ (D) M. A. Lobato, ${ }^{2}$ F. J. Higuera, ${ }^{3}$ I. G. Loscertales, ${ }^{2}$ and A. Fernandez-Nieves ${ }^{4}$

\author{
AFFILIATIONS \\ ${ }^{1}$ Department of Chemistry and Physics, Augusta University, Augusta, Georgia 30912, USA \\ ${ }^{2}$ Department of Ingeniería Mecánica, Térmica, y de Fluidos, Universidad de Málaga, Málaga, Spain \\ ${ }^{3}$ ETSIAE, Universidad Politécnica de Madrid, Madrid, Spain \\ ${ }^{4}$ School of Physics, Georgia Institute of Technology, Atlanta, Georgia 30332, USA \\ ${ }^{5}$ Department of Condensed Matter Physics, University of Barcelona, 08028 Barcelona, Spain \\ ${ }^{6}$ ICREA-Institució Catalana de Recerca i Estudis Avançats, 08010 Barcelona, Spain
}

\begin{abstract}
We apply an electric field to a moderately conducting liquid surrounded by another co-flowing liquid, all inside a glass-based microfluidic device, and study and classify the resulting emission modes. The viscosity and flow rate of the co-flowing liquid affect the number of modes observed in classical electrospray as well as their geometrical characteristics. In particular, we observe a two-dimensional whipping mode not described before. We also provide a qualitative description of some of the modes, including dripping, electrodripping, microdripping, the cone-jet mode, and both the two- and three-dimensional whipping modes.
\end{abstract}

Published under license by AIP Publishing. https://doi.org/10.1063/1.5121714

\section{INTRODUCTION}

Electrospray is a widely used technique to generate droplets. It is based on the fact that a meniscus of a liquid with finite electrical conductivity in the presence of a strong electric field can deform and eventually adopt a conical shape (i.e., the Taylor cone) resulting from the balance between electric and surface tension stresses. ${ }^{1}$ The electric field induces an electric current in the liquid that promotes the accumulation of electric charge at its surface and causes an electric stress that elongates the meniscus in the direction of the field. Under different conditions, the meniscus may either shed charged drops or emit one or several jets which in turn break into drops. Some papers have been published summarizing the observed spraying modes. ${ }^{2-}$ Although it is well known that the atomization process is governed by the physical properties of the liquid, the capillary diameter, electric potential difference, and liquid flow rate, the precise way these quantities affect the generation of emission modes is not completely understood.

Depending on the values of these parameters, several different modes are obtained, emitting aerosols of different characteristics. The modes observed can be classified in two large categories: modes featuring a pulsating meniscus or a steady meniscus. The latter situation, which is stable within certain values of the applied voltage and imposed liquid flow rate, is the workhorse of electrospray and all its associated applications. ${ }^{10-13}$ Following the classification of Jaworek and Krupa, the modes with pulsating meniscus are dripping, microdripping, spindle, multispindle, and ramified meniscus. Meanwhile, for a steady meniscus, the observed modes are oscillating jet, precession, cone-jet, whipping, multijets, and ramified jets. The transition between the last pulsating mode and a steady mode is reached when the electrical stresses equilibrate with the capillary stresses in most of the meniscus. This happens when the liquid used acts as a perfect conductor and the generated steady meniscus is considered hydrostatic, resulting in the well known Taylor field, $E_{T}=\left(2 \gamma / \epsilon \epsilon_{o} d_{t i p}\right)^{1 / 2}$, where $\gamma$ is the liquid surface tension, $\epsilon$ is the dielectric constant of the surrounding dielectric medium, $\epsilon_{o}$ is the vacuum permittivity, and $d_{\text {tip }}$ is the diameter of the tube hosting the Taylor cone. This balance neglects the influence of the inner flow rate, the finite electric conductivity, and the viscosity of the liquid, as well as the effects of a viscous moving outer medium.

In our experiments, the electrical and hydrodynamic stresses are coupled in a co-flow microfluidic device, ${ }^{14}$ similar to that used for generating double emulsions. ${ }^{15}$ The main difference with classical electrospray is that the liquid is ejected into another liquid, as done in Ref. 16, but in our case, this liquid is also flowing. The presence of the outer fluid provides two new experimental parameters we can control, the viscosity and flow rate of 
the outer liquid. These two new variables may change the geometric characteristics of the emission modes and the parametric window where these modes are found in classical electrospray experiments.

In this paper, we present a qualitative study of jet structure and droplet formation in the different electrohydrodynamic spraying regimes when the hosting media is a flowing dielectric liquid. This study allows introducing a classification of spraying modes according to geometric criteria characterizing the shape of the meniscus and the jet. This classification of the functioning regimes is partially based on that introduced in Refs. 3 and 7 even though their classification is proposed for inviscid and highly conductive liquids ejected in air. Among our findings, we report a completely new twodimensional whipping mode, where the oscillations of the jet are confined to a plane.

The structure of the paper is as follows. Section II introduces the experimental setup, the fluids we have used, and how the different measurements are performed. Section III introduces how the state diagrams used to classify the different modes are experimentally obtained, whereas Sec. IV defines the modes themselves, including the novel two-dimensional version of the traditional three-dimensional whipping mode. Section $V$ discusses the transition from pulsating to steady modes, and Sec. VI summarizes our results and concludes.

\section{EXPERIMENTAL SETUP}

We use a microfluidic device consisting of a glass capillary with a square cross section of inner side $a=2 \mathrm{~mm}$ and two cylindrical glass capillaries with similar outer diameter, coaxially aligned with the square one. One of the two cylindrical capillaries is tapered into a tip with inner diameter $d_{t i p}=47 \mu \mathrm{m}$. We pump a liquid with electrical conductivity $K$ and viscosity $\mu_{i}$ through the inside of this capillary, and a dielectric liquid with viscosity $\mu_{o}$ and permittivity $\epsilon=2.1$ through the voids left between the outer square cross section and the inner circular cross section of this capillary, as shown in Fig. 1. These two liquids are immiscible, forming an interface whose interfacial tension is $\gamma=17.5 \times 10^{-3} \mathrm{~N} / \mathrm{m}$. The third liquid, termed liquid collector in Fig. 1, is immiscible with the dielectric liquid, and it is also an electrical conductor that flows through the inside of the second cylindrical capillary, countercurrent to the dielectric liquid. The steady liquid-liquid interface between the dielectric liquid and the liquid collector formed at the exit of the second capillary tube acts as the counter electrode. The exit of the device is provided by the voids left between the outer square

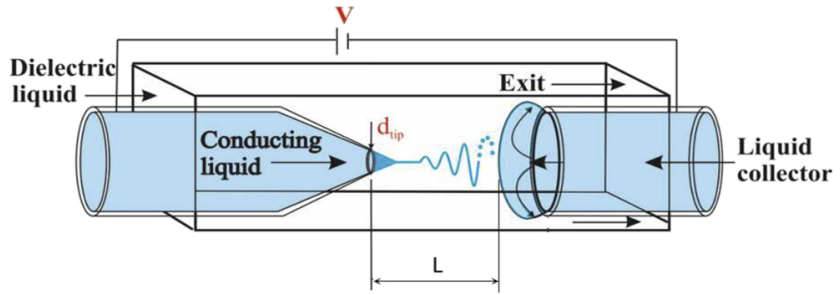

FIG. 1. Schematic of the electro co-flow device.

cross section and the circular cross section of this second cylindrical capillary, as also shown in Fig. 1. Table I summarizes the properties of the liquids we use. To apply the external electric field, we establish an electric potential difference between the metallic needles in contact with the inner and third liquids, which due to their large electrical conductivity act as electrodes. The voltage difference is set by a high-voltage power supply (ACOPIAN P030HP1). Therefore, for a given inner dielectric liquid pair, that is, for $\left(\mu_{i} / \mu_{o}, K\right.$, $\gamma, \epsilon)^{17}$ fixed, the controlling parameters are the flow rates of the dielectric and inner liquids, $q_{o}$ and $q_{i}$, respectively, and the applied voltage, $V$.

Using this electro co-flow configuration, we fix the flow rate of the dielectric liquid to $q_{o}$ and adjust the flow rate of the third liquid to maintain a constant tip-counter-electrode distance $L=0.9 \mathrm{~mm}$. We then vary $q_{i}$ and $V$ to obtain different modes. Both the inner and outer flow rates have been forced through the capillaries by means of pressurized tanks and pressure regulators (4ZM11, Speedaire). The device is placed under a microscope (Axio-Observer.A1, Carls Zeiss) hooked to a high-speed camera (Phantom v7.1, Vision Research) to capture and record the dynamics of the modes. The videos are analyzed with custom software to extract the parameters of interest, including the emission frequencies (for nonsteady modes), the jet oscillation frequencies (for whipping modes), and any other geometric feature that might be of interest. These features are used to identify the different modes.

\section{STATE DIAGRAMS}

We map the ranges of control parameters where each mode is experimentally observed in state diagrams. These diagrams are generated by fixing $\left(\mu_{i} / \mu_{o}, K, \gamma, \epsilon\right)$ for the inner/dielectric liquid pair and sweeping the governing parameters, $q_{i}, q_{o}$, and $V$. The experiments are conducted by fixing the flow rates of the inner and dielectric

TABLE I. Physical properties of the liquids used in the experiments.

\begin{tabular}{lccc}
\hline \hline Liquid & Kinematic viscosity $(\mathrm{cSt})$ & Density $\left(\mathrm{kg} / \mathrm{m}^{3}\right)$ & Conductivity (S/m) \\
\hline Ethylene glycol (EG) & 17 & 1113 & $10^{-4}$ \\
Glycerol & 1118.6 & 1126 & $3 \times 10^{-6}$ \\
PDMS oil I & 10 & 935 & $\ldots$ \\
PDMS oil II & 0.65 & 761 & $\ldots$ \\
PDMS oil III & 50 & 970 & $\ldots$ \\
PDMS oil IV & 20 & 950 & $\ldots$ \\
\hline \hline
\end{tabular}



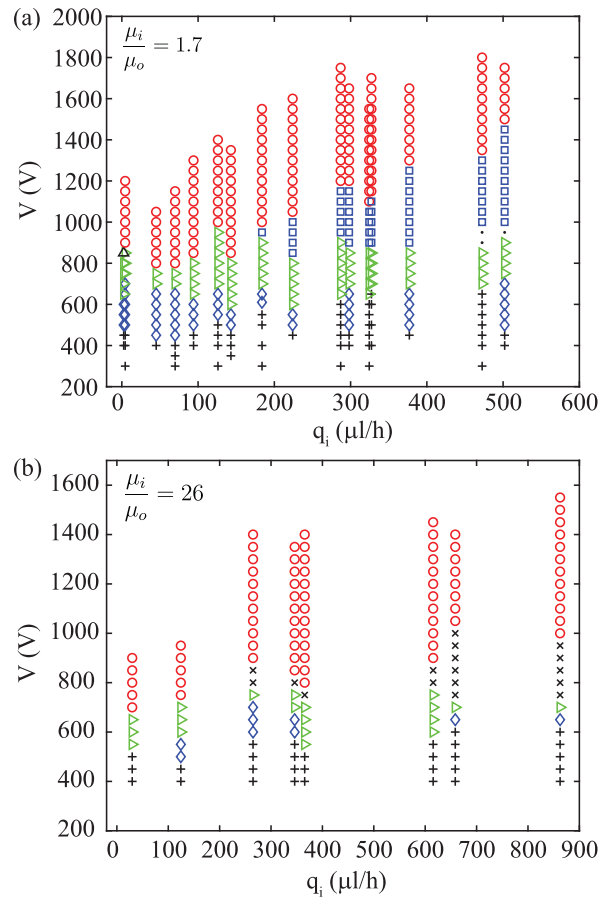

FIG. 2. (a) We use EG for the inner and collector liquids and PDMS oil I as outer liquid at $q_{0}=3 \mathrm{ml} / \mathrm{h}$. (b) EG is used as inner and collector liquids and PDMS oil II for the outer liquid at $q_{0}=16 \mathrm{ml} / \mathrm{h}$. Symbols: +: electrodripping, $\diamond$ : microdripping, $\triangleright$ : spindle, $\circ: 3 D$ whipping, $\square: 2 D$ whipping, and $x$ : long cone-jet.

liquids, $q_{i}$ and $q_{o}$, progressively increasing the applied voltage, $V$, and keeping $L$ constant. The upper limit in the applied voltage is reached when the inner liquid starts receding into the capillary.

Figure 2 shows two state diagrams, each for a given value of the viscosity contrast and outer-fluid flow rate; Fig. 2(a) corresponds to $\mu_{i} / \mu_{o}=1.7$ and $q_{o}=3 \mathrm{ml} / \mathrm{h}$, and Fig. 2 (b) corresponds to $\mu_{i} / \mu_{o}$ $=26$ and $q_{o}=16 \mathrm{ml} / \mathrm{h}$. At low applied voltages, modes with pulsating meniscus are observed, whereas at higher voltages, modes with steady meniscus are seen, including cone-jet, whipping, and a two-dimensional whipping mode not reported before in the literature. Although these modes have resemblance with those observed in electrospray, their features are affected by the presence of the dielectric liquid.

\section{CLASSIFICATION OF THE EMISSION MODES}

\section{A. Pulsating modes}

Following the notation of Jaworek and Krupa, the modes we observe when the meniscus is pulsating are dripping, ${ }^{18}$ electrodripping, ${ }^{19}$ microdripping, ${ }^{20}$ and a spindle mode. These modes have also been classified differently. For example, Juraschek and Rollgen describe the modes in terms of the periodicity of their emission and define an axial mode I, which is characterized by a nonperiodic emission that corresponds to microdripping at low $q_{i}^{7,20}$ and an axial mode II, which is periodic and corresponds to microdripping at high $q_{i}$ and to the spindle mode.

\section{Electrodripping}

In the absence of applied voltage, a drop forms at the exit of the injection capillary in the dripping mode. ${ }^{18}$ Note, however, that when the inner flow rate is moderate or high, the size of the drop can become comparable to or larger than the side-length of the square cross section of the outer capillary and confinement effects can be significant. Nevertheless, the application of an external voltage can eventually result in a decreasing drop size, as shown in Fig. 3, consistent with prior experiments. ${ }^{19}$ To understand this, recall that we enter the electrodripping regime as soon as viscous and electric forces become comparable. Since the electric field at the surface of the meniscus can be expected to be of order of $V / d_{d r o p}$, the electric force would then be of order of $\epsilon \epsilon_{0} V^{2}$. In addition, in the absence of inertia, the viscous force exerted by the inner liquid on the surface of the meniscus is of order of $\mu_{i} q_{i} / d_{d r o p}$, while that of the outer liquid is of order of $\mu_{o} q_{i} / d_{d r o p}$ since, in general, $q_{i} / d_{t i p}^{2}>q_{o} / a^{2}$. Electric and viscous forces would be of the same order if, roughly, $d_{d r o p} / d_{t i p}$ $\sim\left(\mu_{i}+\mu_{o}\right) q_{i} /\left(\epsilon \epsilon_{0} V^{2} d_{t i p}\right)$. In the presence of this balance, $d_{\text {drop }}$ would decrease with increasing $V$ as observed experimentally. Note also that the electric force must necessarily be of the order of the surface tension force, which is of order of $\gamma d_{t i p}$, implying the electric Bond number $B_{E}=\epsilon \epsilon_{0} V^{2} /\left(d_{t i p} \gamma\right)=O(1)$. This condition restricts the occurrence of pulsating modes to a rather narrow range of applied voltages.

\section{Microdripping}

As the voltage increases even further at a constant $q_{i}$, the drop size keeps decreasing until it reaches a size smaller than the capillary tip. Under these conditions, the electrodripping mode transitions to microdripping. For inviscid and highly conductive liquids, this mode has been observed for electric Bond numbers $B_{E}$ approaching one. In this mode, the evolving meniscus is shaped as a sharp conical tip during part of the oscillation cycle, and its emission consists in a drop with a diameter smaller than the tip size together with a small spray. Both these observations are consistent with having an

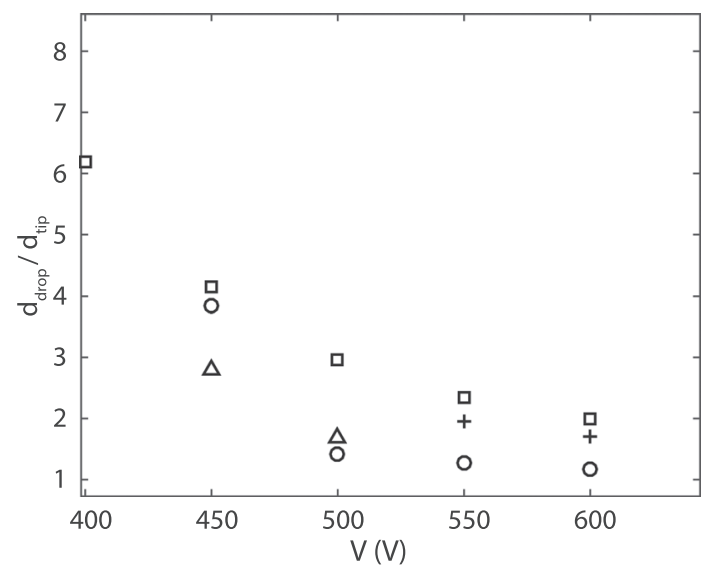

FIG. 3. Dimensionless drop size for the electrodripping mode as a function of the applied voltage. We used EG in PDMS oil I (squares: $q_{i}=396 \mu / \mathrm{h}$ and $q_{0}=100$ $\mathrm{ml} / \mathrm{h}$; pluses: $q_{i}=340 \mu \mathrm{l} / \mathrm{h}$ and $q_{0}=17 \mathrm{ml} / \mathrm{h}$ ) and EG in PDMS oil II (triangles: $q_{i}=346 \mu \mathrm{l} / \mathrm{h}$ and $q_{0}=17 \mathrm{ml} / \mathrm{h}$; circles: $q_{i}=232 \mu \mathrm{l} / \mathrm{h}$ and $q_{0}=30 \mathrm{ml} / \mathrm{h}$ ) 


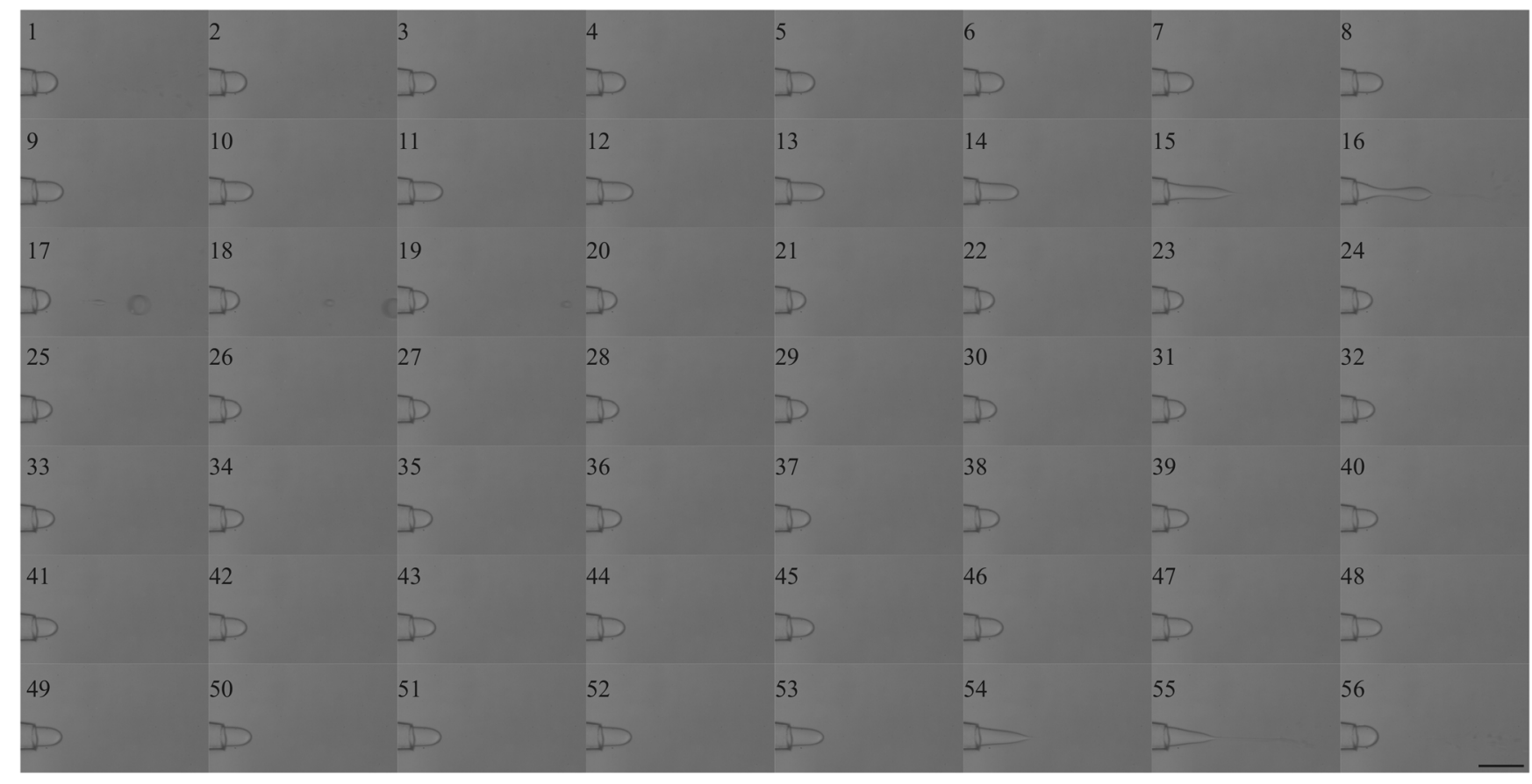

FIG. 4. Snapshot sequence showing aperiodic microdripping for $V=550 \mathrm{~V}, q_{0}=30 \mathrm{ml} / \mathrm{h}$, and $q_{i}=16 \mu / \mathrm{h}$ (EG in PDMS oil II). Time between frames: $200 \mu$ s. The scale bar corresponds to $100 \mu \mathrm{m}$ and is applicable to all panels.

increased surface charge density on the meniscus as $B_{E}$ increases: when $B_{E} \sim 1$, the surface charge density, $\sigma$, may approach the Rayleigh limit, where it becomes $\sigma_{R}$, and the meniscus may become electrically unstable, thus leading to the formation of conical tips, as those observed in microdripping in air. In this latter case, the emission is an aperiodic phenomenon at low $q_{i}^{7,20,21}$ and a periodic one at higher $q_{i}$, with an emission frequency which scales with the inverse of the capillary time $t_{c}=\left(\rho d_{\text {tip }}^{3} / \gamma\right)^{1 / 2}$, varying with the applied voltage as $f t_{c} \sim B_{E}^{1 / 2} d_{t i p}^{3} / V_{m}$, where $V_{m}$ is the volume of the meniscus averaged during the time of one oscillation. ${ }^{20}$ In the case of microdripping in our electro co-flow device, the mode exhibits some of the

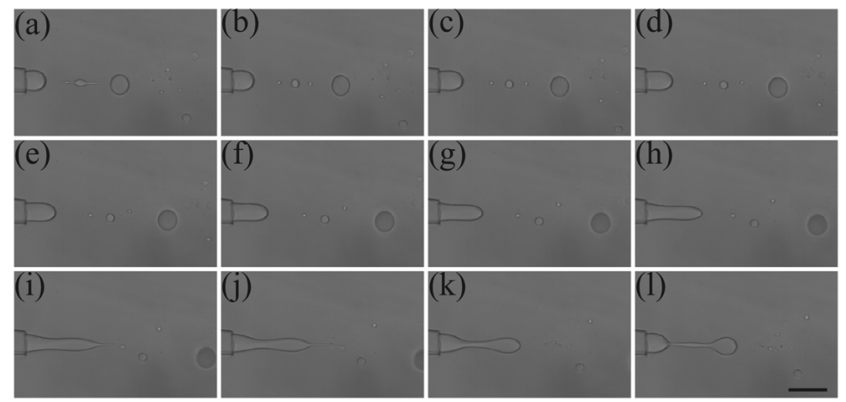

FIG. 5. Snapshot sequence showing periodic microdripping for $V=500 \mathrm{~V}, q_{0}=5$ $\mathrm{ml} / \mathrm{h}$, and $q_{i}=94 \mu \mathrm{l} / \mathrm{h}$ (EG in PDMS oil l). The time between consecutive frames is $200 \mu \mathrm{s}$. The emission period is $\sim 1.2 \mathrm{~ms}$. The scale bar corresponds to $100 \mu \mathrm{m}$ and is applicable to all panels. characteristics we just described albeit not during all the operational window where it exists. Furthermore, the emission frequencies are notably smaller than those measured in air. We find two regions, one aperiodic for low $q_{i}$, shown in Fig. 4, and a periodic one for higher $q_{i}$, shown in Fig. 5, as found in air. The size of the emitted drop is not small anymore at high $q_{i}$, and it does not always coincide with the formation of a conical tip leading to the emission of a spray; see Fig. 6. This makes it hard to distinguish between electrodripping and microdripping. In addition, the frequency seems to be dominated by the presence of the outer fluid rather than by the capillary pressure.

The period of the meniscus oscillation is very large compared to the electric relaxation time of the inner liquid (about $3 \mu$ s for ethylene glycol) and very small compared to the electric relaxation time

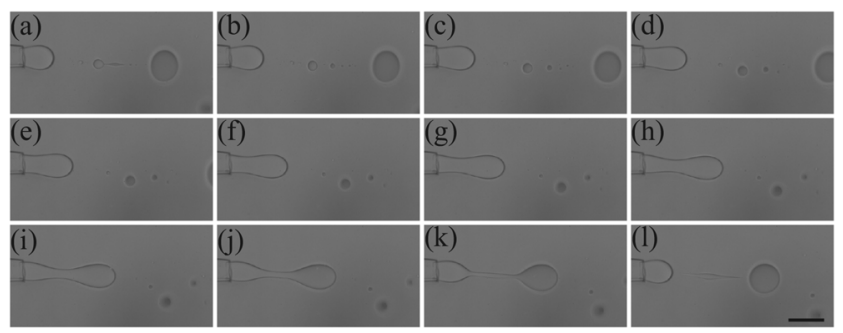

FIG. 6. Snapshot sequence showing periodic microdripping for $V=600 \mathrm{~V}, q_{0}=17$ $\mathrm{ml} / \mathrm{h}$, and $q_{i}=392 \mu \mathrm{l} / \mathrm{h}$ (EG in PDMS oil I). Time between frames: $200 \mu \mathrm{s}$. The scale bar corresponds to $100 \mu \mathrm{m}$ and is applicable to all panels. 
of the outer liquid. In these conditions, electric relaxation effects are confined to a tiny region around the tip that need not be analyzed here, and the meniscus can be taken to be an equipotential mass of liquid (at the applied voltage) immersed in an immiscible dielectric liquid. The effect of inertia is not important in either liquid.

The experiments show that the meniscus is slender during most of the oscillation cycle. Let $\ell(t)$ and $r_{s}(x, t)$ be the length of the meniscus and the local radius of its cross section, where $t$ and $x$ are time and axial distance from the tip of the injection tube. The orderof-magnitude of these variables is denoted $\ell_{c}$ and $r_{c} \ll \ell_{c}$ in what follows. The oscillation period is also large compared to the viscous times $t_{v i}=\rho_{i} r_{c}^{2} / \mu_{i}$ and $t_{v o}=\rho_{i} r_{c}^{2} / \mu_{o}$ of the inner and outer liquids so that the flow of both liquids is dominated by viscosity and the momentum equation reduces to a balance of pressure and viscous forces in each liquid. In these conditions, slender body theory for the Stokes equation ${ }^{22}$ gives an instantaneous, nonlocal relation between the axial velocity of the liquids at the interface, $v_{s}(x, t)$, and the axial force $F(x, t)$ of the meniscus (for $x>0$ ) and the tube (for $x<0$ ) on the outer liquid per unit of axial length. Assuming that the velocity $v_{s}$ is large compared with the velocity of the outer liquid due to its imposed flow rate (of order $q_{o} / a^{2}$ ) and leaving out a factor of the order of the logarithm of the aspect ratio $r_{c} / \ell_{c}$, this relation reduces to $v_{s} \sim F /\left(4 \pi \mu_{o}\right)$ to leading order in an expansion in powers of the logarithm of $r_{c} / \ell_{c}$.

Integrating the momentum equation for the inner liquid across the section of the meniscus and using the boundary conditions at its surface, we obtain the balance of forces

$$
\pi \gamma \frac{\partial r_{s}}{\partial x}+\pi r_{s}^{2} \frac{\partial \tau_{e}}{\partial x}+2 \pi r_{s} \tau_{v}=0
$$

where $\tau_{e}=\epsilon \epsilon_{0} E^{2} / 2$, with $E$ being the electric field at the surface, is the electric stress, which is normal to the equipotential liquid surface, and $\tau_{v}$ is the shear stress of the outer liquid on the surface so that $F=-2 \pi r_{s} \tau_{v}$. Here, the axial viscous force in the inner liquid, which is of order $r_{s}^{2} \mu_{i} \partial v_{s} / \partial x$ per unit length of the meniscus, has been left out because it is small compared to the viscous force due to the outer liquid [third term in (1)] when $r_{c} / \ell_{c} \ll 1$.

Balance (1) gives $F$ in terms of the surface tension and electric forces acting on the surface. The first of these is negative when $\partial r_{s} / \partial x<0$ (meniscus without a neck). The surface tension then pushes the inner liquid backwards toward the injection tube and the elongation of the meniscus is entirely due to the electric force [second term of (1)], which therefore plays an important role in the dynamics. However, the electric force cannot be much larger than the surface tension force because a $\tau_{e} \gg \gamma / r_{c}$ would imply that the surface charge density $\sigma=E / \epsilon \epsilon_{0}$ for an equipotential liquid is large compared to $\sigma_{R}$, further implying that the electric stress would overcome the surface tension stress causing the slender meniscus to become unstable. ${ }^{23}$ Finally, the viscous force of the outer liquid on the surface [third term in (1)] must also play a role because a hydrostatic equilibrium of electric and surface tension forces is not possible for a slender meniscus. ${ }^{24}$ Thus, all three terms in (1) must be of the same order.

Surface tension tends to generate a neck on the slender meniscus that eventually undergoes pinch-off and sheds a droplet. The characteristic time of this process, $t_{s}$ say, can be estimated noticing that the radial velocity of the inner liquid is of order $v_{r s}$ $=r_{c} / t_{s}$ and, by continuity, it has an associated axial velocity of order $v_{x s}=v_{r s} \ell_{c} / r_{c}=\ell_{c} / t_{s}$. Assuming that the velocity $v_{s}$ of the liquids at the surface is of order $v_{x s}$, which amounts to saying that $t_{s}$ is of the order of the oscillation period, the balance of the first and third terms in (1) leads to

$$
t_{s}=\frac{\mu_{0} \ell_{c}^{2}}{\gamma r_{c}} .
$$

The electric force [second term in (1)] also plays a role, but a direct estimation of this force is difficult. If the electric field at the surface of the meniscus was only due to the applied voltage $V$, then $E$ $\sim V / r_{c}$ would be expected up to a factor of the order of the logarithm of the aspect ratio. Then, $\tau_{e} \sim \epsilon \epsilon_{0} V^{2} / r_{c}^{2}$ and the balance of the second and third terms in (1) would give a velocity $v_{s} \sim \epsilon V^{2} / \mu_{0} \ell_{c}$. This, however, is of the order of $2.3 \mathrm{~m} / \mathrm{s}$ for $\mu_{0}=9.3 \mathrm{mPa} \mathrm{s}$, $V=700 \mathrm{~V}$, and $\ell_{c}=200 \mu \mathrm{m}$, which are typical values in the microdripping regime. The estimated $v_{s}$ is large compared to the measured elongation velocity of the meniscus. The discrepancy points out that the space charge due to previously detached droplets that drift slowly in the outer liquid significantly decreases the electric field and the electric stress on the meniscus. While an accurate estimation of the space charge is beyond the scope of this analysis, the comment above on the importance of the three terms in (1) suggests that the electric stress on the partially screened surface of the meniscus is of the order of the surface tension stress and that the estimation (2) remains valid.

The condition that the volume of each emitted droplet, of order $\ell_{c} r_{c}^{2}$, should coincide with the volume of inner liquid injected during an oscillation period reads

$$
q_{i} t_{s} \sim \ell_{c} r_{c}^{2} .
$$

Conditions (2) and (3) must be supplemented with an additional relation to close the problem and estimate $\ell_{c}, r_{c}$, and $t_{s}$. We can obtain this relation from the experiment by noting that the length and maximum radius of the ligament immediately before detachment of a droplet are approximately proportional to each other (see Fig. 7). This implies

$$
\ell_{c} \approx k r_{c}
$$

with $k=10-15$ from the experimental results.

$$
\text { Combining (2)-(4), we obtain }
$$

$$
\ell_{c} \sim k^{3 / 2}\left(\frac{\mu_{o} q_{i}}{\gamma}\right)^{1 / 2}, \quad r_{c} \sim k^{1 / 2}\left(\frac{\mu_{o} q_{i}}{\gamma}\right)^{1 / 2}, \quad t_{s}^{-1} \sim \frac{1}{k^{5 / 2}}\left(\frac{\gamma^{3}}{\mu_{o}^{3} q_{i}}\right)^{1 / 2},
$$

where $t_{s}^{-1}$ is an estimation of the frequency of the meniscus oscillation in the microdripping regime.

The absence of the applied voltage $V$ in (5) does not mean that electric forces are negligible. Instead, the microdripping regime requires that the electric field of the space charge nearly cancels the field of the applied voltage on the meniscus. The relatively narrow range of $B_{E}$ where this regime is realized seems to reflect that this approximate cancellation is possible only under very specific conditions. The oscillation frequency and other features of the flow depend on $B_{E}$ in the narrow range of microdripping, showing that the extent of the cancellation changes within this range. This translates into a dispersion of the experimental data about the estimations (5), shown in Fig. 8, which do not account for such fine details. 

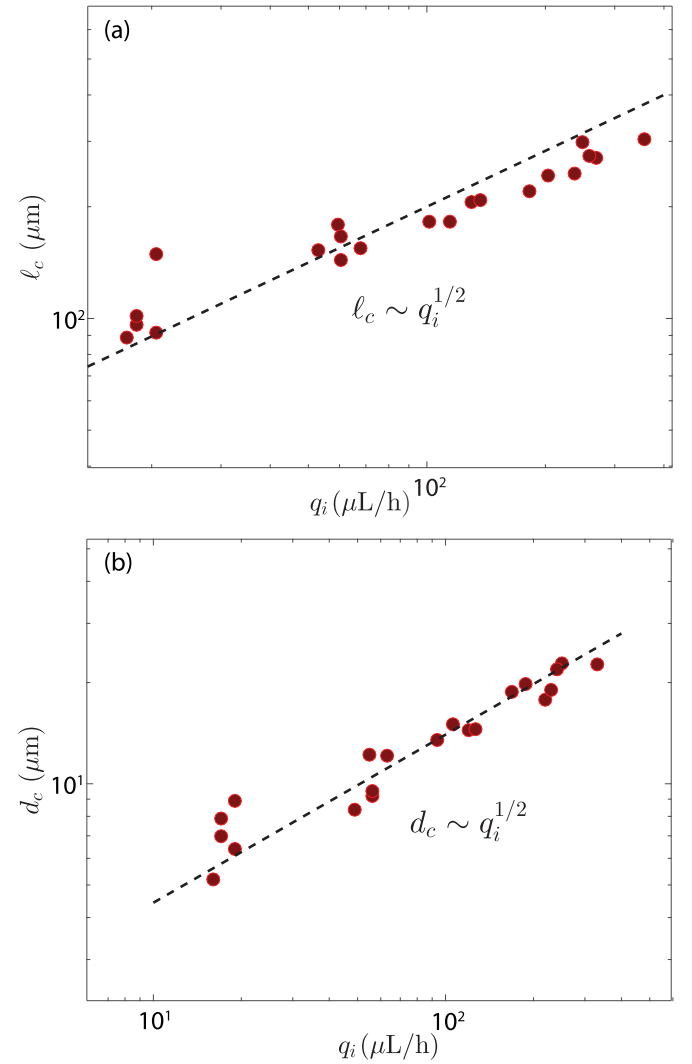

FIG. 7. (a) Length of the ligament $\ell_{c}$, before the detachment from the meniscus, with respect to the flow rate of the inner liquid $q_{i}$; the dashed straight line has slope $1 / 2$, and (b) mean diameter of the ligament $d_{c}$, before detachment from the meniscus, with respect to the flow rate of the inner liquid $q_{i}$. The dashed straight line has slope of $1 / 2$.

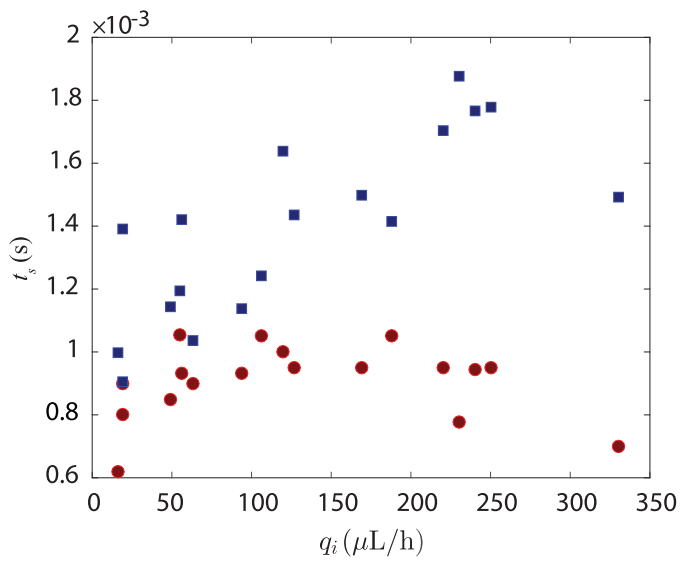

FIG. 8. Ligament formation time, $t_{s}$, vs inner flow rate, $q_{i}$, for $\mu_{0}=9.3 \times 10^{3} \mathrm{~Pa} \mathrm{~s}$, $V=700 \mathrm{~V}$, and $d_{t i p}=50 \mu \mathrm{m}$. Circles correspond to experimental values. Squares correspond to values from Eq. (2). Results corresponding to different outer flow rates are represented in this figure, since $q_{0}$ has no meaningful effect in oscillating regimes.

\section{Spindle mode}

If we keep increasing the applied voltage, while still $B_{E} \sim 1$, we reach the spindle mode, where the meniscus is stretched and becomes much longer than $d_{\text {tip }}$. It differs from the microdripping mode in that no regular droplets are ejected from the meniscus but only elongated fragments of liquid. After its detachment, each fragment can further break into several smaller droplets of different sizes, while the meniscus recoils to its initial shape, which in contrast with the previous modes, is somehow more conical than rounded. At that point, a new thread starts to form. The large surface charge on the elongated meniscus could also cause it to whip slightly before it breaks into droplets; see Fig. 9. At higher voltages, the transition to a steady meniscus happens.

\section{B. Steady modes}

In the literature, the cone-jet and whipping modes are the most studied ones.

\section{Cone-jet mode}

The cone-jet mode is an axisymmetric mode where the meniscus is stretched into a conical shape; the singularity of its tip is in reality a small region where the cone transitions to a thin jet that eventually breaks into highly charged droplets. This mode was denoted by Juraschek and Rollgen ${ }^{8}$ as Axial mode III. It is found at low $q_{i}$, and it has been greatly used to generate droplets with a size much smaller than the tip size. At high $q_{i}$, a mode reminiscent to cone-jet is observed; it is a cone-jet with a longer and thicker jet than the classical one. ${ }^{16}$ It is worth making this distinction for three reasons: (i) these modes appear for different experimental conditions, low and high $q_{i}$, (ii) the shapes of the menisci are different, being convex at low $q_{i}$ and concave at high $q_{i}$ (see Fig. 10) and, (iii) the nature of this mode is different; the classical cone-jet comes from a balance between capillary and electrical stresses, while the long jets are purely hydrodynamic modes, coming from the competition between capillary and viscous stresses, as we will discuss at the end of Sec. IV.

\section{Whipping: 3D and 2D versions}

The whipping mode is a non axisymmetric mode where the jet violently slashes before breaking into droplets. The geometric
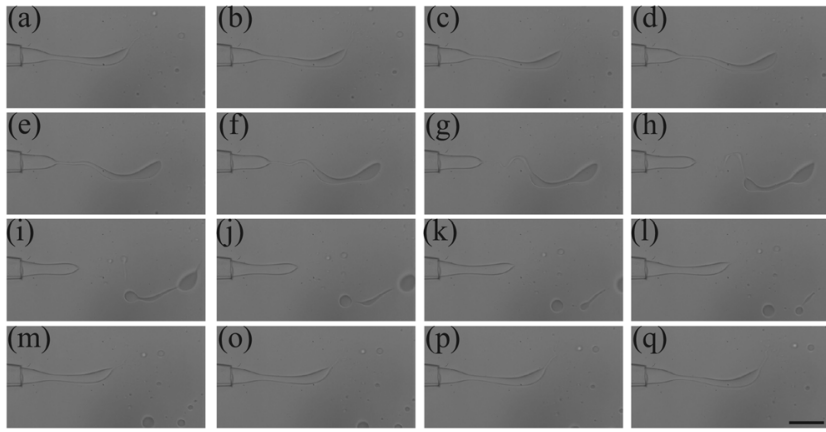

FIG. 9. Snapshot sequence showing the spindle mode for $V=850 \mathrm{~V}, q_{0}=17 \mathrm{ml} / \mathrm{h}$ and $q_{i}=202 \mu \mathrm{l} / \mathrm{h}$ (EG in PDMS oil I). Time between frames: $100 \mu \mathrm{s}$. The scale bar corresponds to $100 \mu \mathrm{m}$ and is applicable to all panels. 


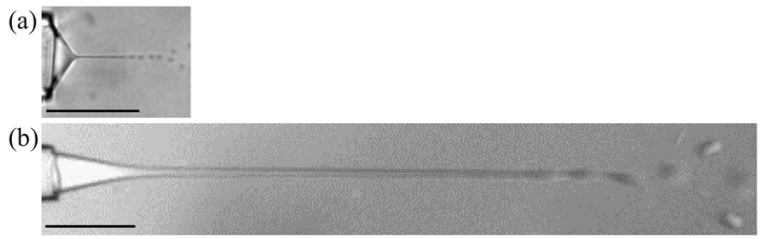

FIG. 10. Cone-jet mode. (a) EG in PDMS oil I at $q_{0}=3 \mathrm{ml} / \mathrm{h}, q_{i}=30 \mu \mathrm{l} / \mathrm{h}$, and $\mathrm{V}=660 \mathrm{~V}$. (b) EG in PDMS oil II at $q_{0}=30 \mathrm{ml} / \mathrm{h}, q_{i}=232 \mu \mathrm{l} / \mathrm{h}$, and $\mathrm{V}=750 \mathrm{~V}$. The scale bar corresponds to $100 \mu \mathrm{m}$.

structure of the jet in this mode depends on the outer medium, having a helicoidal shape [see Fig. 11(a) and video 1 (Multimedia view)] when the longest characteristic time of the system is associated with the outer liquid ${ }^{25}$ or being chaotic [Fig. 11(b) and video 2 (Multimedia view)] when the time scale associated with the outer fluid is smaller or of the same order than that of the inner fluid. This whipping mode, when performed in air or vacuum, is the basis of all electrospinning techniques.

Importantly, we have observed a new mode, which we call 2D-whipping [see Fig. 12 and video 3 (Multimedia view)]. It is a nonaxisymmetric mode where the jet oscillates as in the whipping mode but with the oscillation confined to a plane. When the applied voltage is increased, this plane also oscillates and eventually rotates leading to the conventional three-dimensional whipping. Similar modes were observed by $\mathrm{Cubaud}^{26}$ using miscible liquids in a two-dimensional flow-focusing device without any applied electric forces.

When the whipping structure is well defined, one can quantify some of its geometrical properties and see the differences between

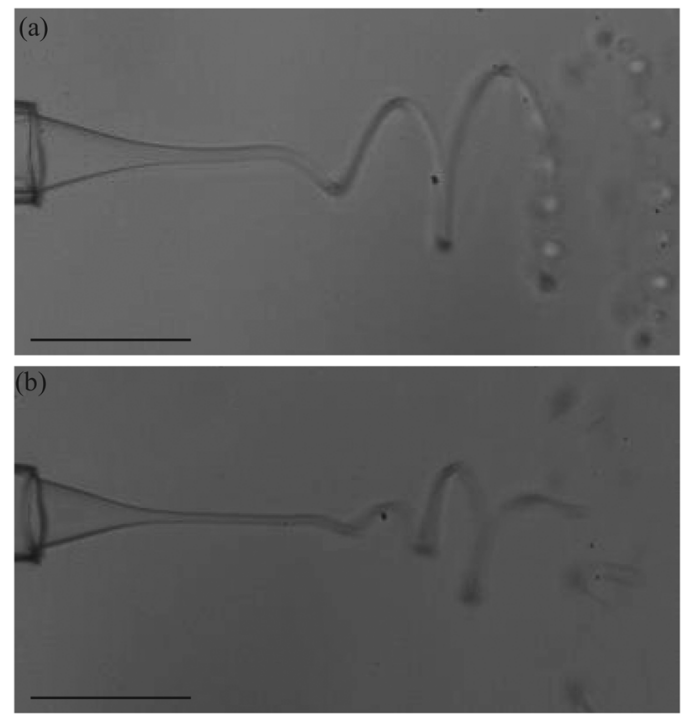

FIG. 11. (a) Helicoidal whipping mode of EG in PDMS oil I at $V=1050 \mathrm{~V}$ $q_{0}=100 \mathrm{ml} / \mathrm{h}$, and $q_{i}=41 \mu \mathrm{l} / \mathrm{h}$. (b) Chaotic whipping mode of EG in PDMS oil II at $V=750 \mathrm{~V}, q_{0}=16 \mathrm{ml} / \mathrm{h}$, and $q_{i}=125 \mu \mathrm{l} / \mathrm{h}$. The scale bar corresponds to $100 \mu \mathrm{m}$. Multimedia views: (a) https://doi.org/10.1063/1.5121714.1; (b) https://doi.org/10.1063/1.5121714.2
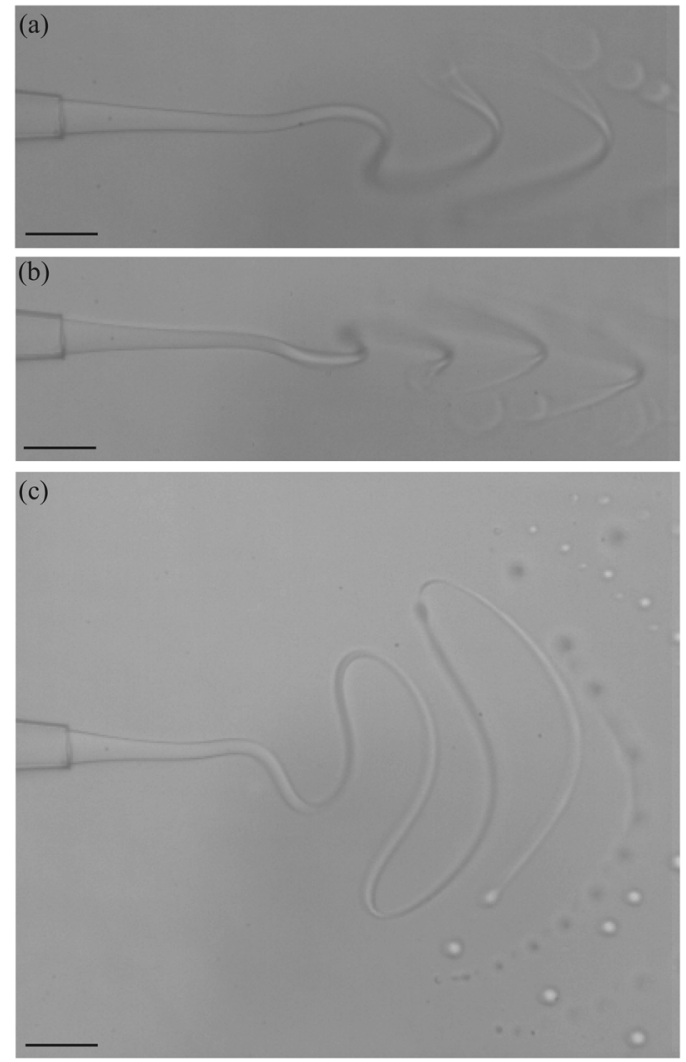

FIG. 12. Evolution of the 2D-whipping with applied voltage for EG in PDMS oil I with $q_{0}=16 \mathrm{ml} / \mathrm{h}, q_{i}=392 \mu \mathrm{l} / \mathrm{h}$, and (a) V = $1050 \mathrm{~V}$, (b) $\mathrm{V}=1150 \mathrm{~V}$, or (c) $V=1250 \mathrm{~V}$. The scale bar corresponds to $100 \mu \mathrm{m}$. Multimedia view: (a) https://doi.org/10.1063/1.5121714.3

the two- and three-dimensional versions of the whipping mode. Figure 13 shows the frequency dependence of the whipping modes with the capillary number associated with the inner liquid for different applied voltages [Fig. 13(a)] and outer viscosities [Fig. 13(b)]. The frequency, $F$, decreases with the inner flow rate, flattening up where the transition to the $2 \mathrm{D}$-whipping mode happens. The outer flow rate does not seem to play a role, while the applied voltage increases the frequency associated to $3 \mathrm{D}$-whipping. The frequency of the 2D-whipping is independent of all these variables, reinforcing the idea that its nature is hydrodynamic, similar to the observations in Ref. 26.

Analyzing the data in Fig. 13 following Ref. 27 allows collapsing these data. The idea behind is that the whipping is caused by an interplay between electric stresses, pulling and pushing laterally the jet, the viscous stresses exerted by the outer liquid as the jet moves, and surface tension stresses, which oppose jet stretching. ${ }^{28}$ The viscous effect due to the outer fluid is caused by the movement of the jet, so it seems appropriate to introduce a mixed capillary number $C a_{i o}=\mu_{o} q_{i} / \gamma d_{\text {tip }}^{2}$, in which the velocity is that of the jet interface, which is related to the inner flow rate $q_{i}$. Also, the viscous time due to the outer fluid is important in the dynamics, ${ }^{25} t_{v c}=\mu_{o} d_{t i p} / \gamma$, and so is the electric Bond number, $B_{E}$. Following Ref. 27, we find 

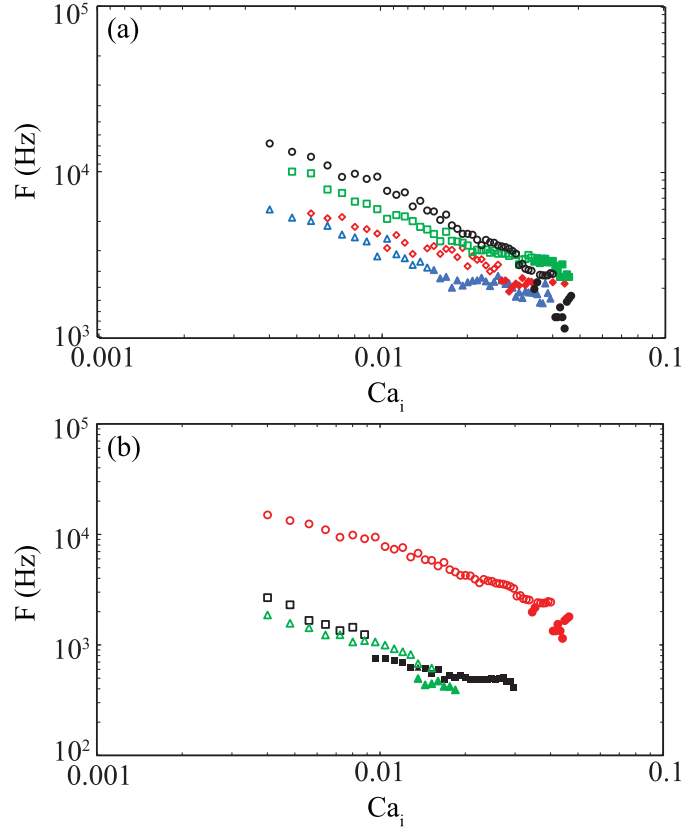

FIG. 13. Frequency for the 3D (empty symbols) and 2D (filled symbols) versions of the whipping mode for (a) different applied voltages and (b) different outer viscosities: (a) EG in PDMS oil I for (triangles) $1000 \mathrm{~V}$, (diamonds) $1050 \mathrm{~V}$, (squares) 1100 V, and (circles) 1150 V. (b) Circles: PDMS oil I. Squares: PDMS oil IV. Triangles: PDMS oil III.

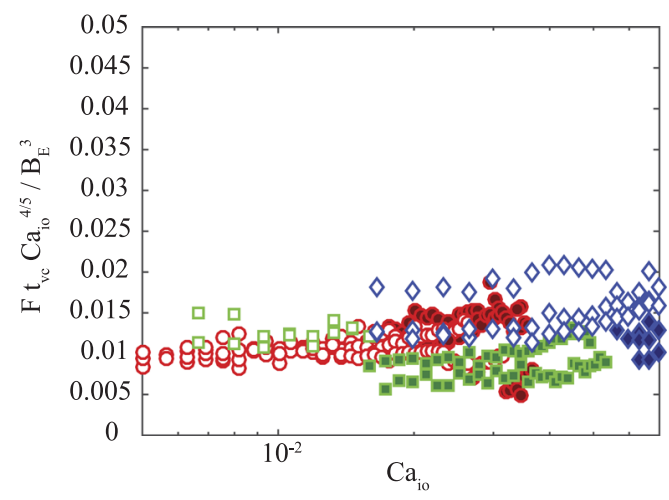

FIG. 14. Dimensionless frequency, $\mathrm{Ft}_{v c} \mathrm{Ca} a_{i o}^{4 / 5} / B_{E}^{3}$, vs the mixed capillary number $C a_{i o}$. Symbols: circles: PDMS oil I, squares: PDMS oil IV, and diamonds: PDMS oil III. Filled symbols: 2D-whipping. Hollow symbols: 3D-whipping.

that for all the data in Figs. 13(a) and 13(b), the dimensionless frequency $F t_{v c} C a_{i o}^{4 / 5} / B_{E}^{3}$ remains practically constant vs $C a_{i o}$, as shown in Fig. 14.

\section{TRANSITION FROM PULSATING TO STEADY MODES}

It seems interesting to inquire further about the conditions in which pulsating modes transition to steady ones. Figure 15 shows the state diagrams for two viscosity ratios and different $q_{o}$ values, illustrating how the latter control parameter does not affect the
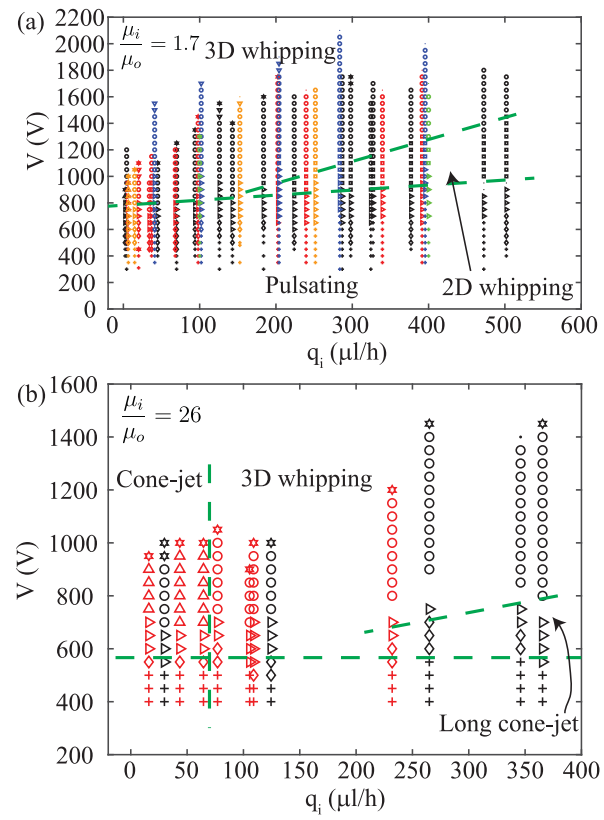

FIG. 15. (a) $z_{\text {Taylor }} / d_{\text {tip }}$ vs $q_{i}$ for $E G$ for the inner and collector liquid and PDMS oil I for the outer liquid for different $q_{0}: 3 \mathrm{ml} / \mathrm{l}$ (black), $17 \mathrm{ml} / \mathrm{h}$ (red), $100 \mathrm{ml} / \mathrm{h}$ (blue), $300 \mathrm{ml} / \mathrm{h}$ (green), and $0.3 \mathrm{ml} / \mathrm{h}$ (orange). (b) $z_{\text {Taylor }} / d_{\text {tip }}$ vs $q_{i}$ for EG for the inner and collector liquid and PDMS oil II for the outer liquid with $q_{0}=16 \mathrm{ml} / \mathrm{h}$ (black) and $30 \mathrm{ml} / \mathrm{h}$ (red). Symbols: +: electrodripping, $\diamond$ : microdripping, $\triangleright:$ spindle, $\circ$ : 3D whipping, $\square: 2 \mathrm{D}$ whipping, and $\times$ : long cone-jet.

parametric region where each mode is observed. According to Ref. 25, this can be explained by looking at the characteristic times in our problem. Indeed, the relevant dimensionless numbers can be conveniently written in terms of the relevant time scales for each of the liquids. Thus, the Reynolds number $R e=\rho v r / \mu=t_{i} / t_{v}$, the Weber number $W e=\rho r v^{2} / \gamma=\left(t_{i} / t_{c}\right)^{2}$, the Ohnesorge number,

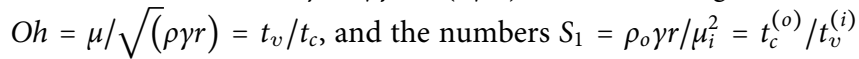
and $S_{2}=\rho_{i} \gamma r / \mu_{o}^{2}=t_{c}^{(i)} / t_{v}^{(o)}$ can all be written in terms of the inertial time $t_{i}=\rho v r^{2} / \gamma$, the viscous time $t_{v}=\mu r / \gamma$, and the capillary time $t_{c}=\left(\rho r^{3} / \gamma\right)^{1 / 2}$, where $\rho$ is the density of the liquid, $v$ is the speed, $r$ is a characteristic length scale, and $(i)$ and $(o)$ refer to inner and outer liquids; $R e, W e$, and $O h$ are defined for both liquids. Only the inertial time depends on the flow rate. If we estimate these numbers for the outer liquid with our range of $q_{o}$ values, the inertial time is the shortest time scale, explaining the lack of $q_{o}$ influence.

Although there are differences between the state diagrams in Fig. 15, in both cases, we can observe a transition from the last pulsating meniscus mode to the first steady-state meniscus mode at an almost constant applied voltage, for any $q_{i}$. Taylor ${ }^{1}$ explained this transition with a balance between the capillary and electric forces in the case of a hydrostatic, perfectly conducting meniscus ejected in air. That balance leads to the well-known "Taylor field," $E_{T} \approx\left(2 \gamma / \epsilon \epsilon_{o} d_{t i p}\right)^{1 / 2}$, which also provides the maximum surface charge density the meniscus of a conducting liquid can withstand, $\sigma_{T} \approx \epsilon \epsilon_{o} E_{T}$. The static equilibrium of the conical meniscus is broken when other stresses become important, resulting in jet formation. 
Since in most of our experiments static equilibrium breaks near the apex of the conical menisci as the electrical relaxation time is short compared to any other relevant time scale, we can safely assume that nearly all our menisci remain equipotential. Therefore, one can assume that when an oscillating mode transitions to a steady mode, the meniscus acquires a charge corresponding to the Taylor electric field. To use the Taylor field to scale our data, a model for the electric field for our geometry is required. Gundabala et al. ${ }^{14}$ used the needle-plate field to model the electric field in electro co-flow, $E_{\text {needle-plate }}=C V / z \ln \left(2 \mathrm{~L} / d_{\text {tip }}\right)$, where $C$ is a geometric factor estimated numerically $(C=0.59)$ and $z$ is an axial distance measured from the tip of the meniscus. If we use $E_{\text {needle-plate }}$ to model the electric field at the transition conditions, we can compute the values of $z$ where $E_{\text {needle-plate }}=E_{T}$; we call this $z_{\text {Taylor }}$. Figure 16 shows the axial distance, $z_{\text {Taylor }}$, made dimensionless with $d_{t i p}$, at the experimental conditions where the transition from pulsating to steady modes occur for different values of $q_{i}$ and $q_{o}$. For all the experiments, we obtain $z_{\text {Taylor }} \approx d_{\text {tip }} / 2$, consistent with the idea that once the Taylor electric field is reached the pulsating mode transitions into a steady-state one.

Using only the modified Taylor model described above to describe the pulsating-to-steady transition is incomplete though because it does not take into account either the flow rate or viscosity of the outer dielectric liquid. As we have seen in Figs. 2 and 15, this transition does not depend on $q_{i}$ nor $q_{o}$, while $\mu_{o}$ affects slightly the voltage $V$ at which the transition takes place, also determining the mode we transition to. Clearly, the ratio $\mu_{i} / \mu_{o}$ affects the transition. Although the details are far beyond the scope of the present work, it is clear that the viscous drag exerted by the dielectric liquid on the meniscus must affect its dynamics. It is not surprising that the voltage $V$ required for the transition from pulsating to steady modes increases with $\mu_{o}$ since as the steady jet develops it drags the outer liquid with it, so the electric pulling must overcome this extra drag to become steady. Furthermore, the stability of such a straight charged jet should also be affected by the outer drag, promoting nonaxisymmetric modes as it increases. This is in qualitative agreement with what is shown in Fig. 15, where, for low values of $q_{i}$, the conejet transitions to 3D whipping, while for large values of $q_{i}$, the long straight jets develop into $2 \mathrm{D}$ whipping. This is also consistent with the idea that some of these modes might be purely hydrodynamic.

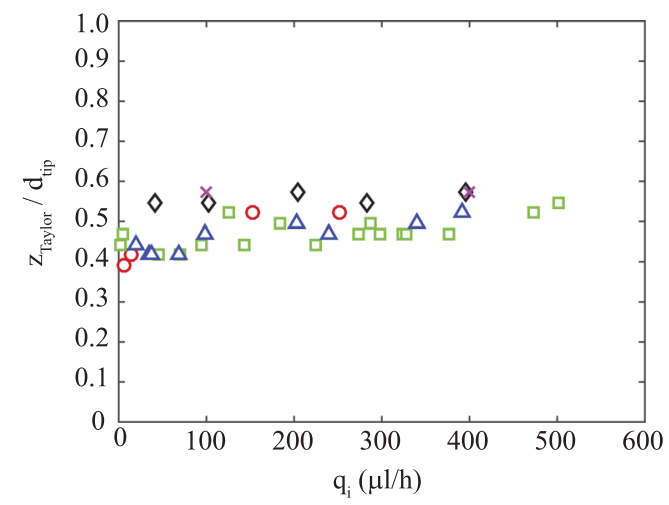

FIG. 16. We used EG in PDMS oil I for different $q_{0}$ : circles: $0.3 \mathrm{ml} / \mathrm{h}$, squares: 3 $\mathrm{ml} / \mathrm{h}$, triangles: $17 \mathrm{ml} / \mathrm{h}$, diamonds: $100 \mathrm{ml} / \mathrm{h}$, and crosses: $300 \mathrm{ml} / \mathrm{h}$.

\section{CONCLUSIONS}

In this paper, we have used the microfluidic device developed in Ref. 14 to experimentally study the different atomization modes that appear in electro co-flow. For a given pair of liquids, that is, for $\left(\mu_{i} / \mu_{o}, K, \gamma, \epsilon\right)$ constant, the controlling parameters are the liquid flow rates, $q_{i}$ and $q_{o}$, and the applied voltage, $V$. In the experiments, $q_{i}$ and $q_{o}$ are fixed, while $V$ is increased. The different modes have been classified based on the description introduced in Ref. 7 .

We have obtained the state diagrams of the observed modes for four different liquid pair combinations. The flow rate of the dielectric liquid, $q_{o}$, plays no role in the qualitative shape of the state diagrams for the range of flows used in this work. We can generally classify the modes in two main categories: steady and unsteady.

The unsteady modes are observed for voltages $V$ smaller than a threshold value. This threshold value seems to be that at which the meniscus attains the Taylor field, $E_{T}$, based on $d_{t i p}$.

For smaller voltages, we observe electrodripping, where drops larger than $d_{t i p}$ detach from the capillary tip dragged by the electric stresses. This situation happens when $B_{E}<1$. The size of the droplets decreases with increasing $V$ and increases with the viscosity of the outer medium.

Microdripping is obtained when $B_{E} \rightarrow 1$ and $B_{E}<1$ and for small and moderate values of $q_{i}$. In this regime, the meniscus develops a sharp tip that emits a fine aerosol and eventually detaches from a stretched meniscus in the form of a droplet. In our experiments, the oscillating frequency, $f$, seems to be dominated by the competition between shear viscous stresses exerted by the outer dielectric fluid on the meniscus and the capillary pressure responsible for the pinchoff of the droplets. This suggests that $f \sim t_{s}^{-1}=\gamma r_{c} /\left(\mu_{o} \ell_{c}^{2}\right)$, with $\ell_{c}$ and $d_{c}$ being the characteristic length and diameter of the ligament detached from the meniscus in each oscillation. Estimates of $\ell_{c}$ and $d_{c}$ are also obtained by imposing mass conservation in the meniscus so that $\ell_{c} \sim q_{i}^{1 / 2}$ and $d_{c} \sim q_{i}^{1 / 2}$. When $q_{i}$ is increased further, while maintaining $V$ in the microdripping regime, one enters into the spindle mode, where the meniscus stretches up to a length substantially larger than $d_{\text {tip }}$. These long liquid ligaments may break into several drops of different sizes and, for higher voltages, may result in whipping.

When the voltage $V$ overcomes a critical value at which the electric field on the meniscus equals $E_{T}$, the system enters into steady regimes, characterized by a steady meniscus, emitting an electrified jet that eventually breaks into charged droplets.

For large values of $\mu_{i} / \mu_{o}$, the electrified jet presents three steady modes. For low values of $q_{i}$, we find steady cone-jets as those observed in regular electrosprays. As the flow rate increases, $3 \mathrm{D}$ whipping appears except for voltages slightly larger than the critical one, where we observe long straight jets that reach the liquid collector unbroken.

For $\mu_{i} / \mu_{o} \leq O(1)$, the steady cone-jet mode is either pushed to values of $q_{i}$ not accessed in our experiments or completely disappears so that only $3 \mathrm{D}$ whipping jets are observed in our experiments, except for large values of $q_{i}$ and at voltages slightly larger than the critical one, where we have discovered a new mode: $2 \mathrm{D}$ whipping. In this regime, the slashing motion of the jet is contained in a plane which contains the axis of the device and, that depending on the voltage, may rotate around it. This new mode is perhaps a purely 
hydrodynamic mode since similar behaviors have been observed for uncharged jets.

The whipping frequencies $F$ for the $2 \mathrm{D}$ and $3 \mathrm{D}$ whipping modes seem to depend on the interplay between viscous stresses of the outer dielectric fluid and the electric stresses on the whipping jet. We experimentally find that $F t_{v c} \sim B_{E}^{3} / C a_{i o}^{4 / 5}$, where $C a_{i o}=\mu_{o} q_{i} /\left(\gamma d_{t i p}^{2}\right)$ and $t_{v c}=\mu_{o} d_{t i p} / \gamma$ is the outer fluid viscocapillary time.

We finally note that we have focused on the qualitative description of the observed modes, including a novel two-dimensional whipping mode, proposing scalings that must be taken as a starting point. More theoretical work is needed to describe and understand the detailed properties of all modes and transitions observed in electro co-flow.

\section{ACKNOWLEDGMENTS}

This work was supported by the NSF (Grant No. DMR1609841), the ACS PRF (Grant No. 60302-UR9), and the Spanish Ministerio de Economía y Competitividad (Grant Nos. DPI201571901-REDT, DPI2017-86547-C2-1-P, and DPI2017-86547-C2-2$\mathrm{P})$. The authors gratefully acknowledge Professor J. M. Gordillo (Universidad de Sevilla) for suggestions.

\section{REFERENCES}

${ }^{1}$ G. Taylor, "Disintegration of water drops in an electric field," Proc. R. Soc. London, Ser. A 280, 383-397 (1964).

${ }^{2}$ I. Hayati, A. I. Bailey, and T. F. Tadros, "Investigations into the mechanisms of electrohydrodynamic spraying of liquids: I. Effect of electric field and the environment on pendant drops and factors affecting the formation of stable jets and atomization,” J. Colloid Interface Sci. 117, 205-221 (1987).

${ }^{3} \mathrm{M}$. Cloupeau and B. Prunet-Foch, "Electrostatic spraying of liquids: Main functioning modes," J. Electrost. 25, 165-184 (1990).

${ }^{4}$ J. M. Grace and J. C. M. Marijnissen, "A review of liquid atomization by electrical means,” J. Aerosol Sci. 25, 1005-1019 (1994).

${ }^{5}$ A. I. Grigorev and S. O. Shiryaeva, "The theoretical consideration of physical regularities of electrostatic dispersion of liquids as aerosols," J. Aerosol Sci. 25, 1079-1091 (1994).

${ }^{6}$ S. O. Shiryaeva and A. I. Grigorev, "The semiphenomenological classification of the modes of electrostatic dispersion of liquids," J. Electrost. 34, 51-59 (1995).

${ }^{7}$ A. Jaworek and A. Krupa, "Main modes of electrohydrodynamic spraying of liquids," in Third International Conference on Multiphase Flow ICMF, 1998.

${ }^{8}$ R. Juraschek and F. W. Rollgen, "Pulsation phenomena during electrospray ionization,” Int. J. Mass Spectrom. 177, 1-15 (1998).
${ }^{9}$ J. Rosell-Llompart, J. Grifoll, and I. G. Loscertales, "Electrosprays in the cone-jet mode: From Taylor cone formation to spray development," J. Aerosol Sci. 125, 2-31 (2018).

${ }^{10}$ A. Barrero and I. G. Loscertales, "Micro- and nanoparticles via capillary flows," Annu. Rev. Fluid Mech. 39, 89-106 (2007)

${ }^{11}$ C. M. Whitehouse, R. N. Dreyer, M. Yamashita, and J. B. Fenn, "Electrospray ionization for mass-spectrometry of large biomolecules," Science 246, 64-71 (1989).

${ }^{12}$ T. J. Sill and H. A. von Recum, "Electrospinning: Applications in drug delivery and tissue engineering," Biomaterials 29, 1989-2006 (2008).

${ }^{13}$ P. P. Tsai, H. Schreuder-Gibson, and P. Gibson, "Different electrostatic methods for making electret filters," J. Electrost. 54, 333-341 (2002).

${ }^{14}$ V. Gundabala, N. Vilanova, and A. Fernandez-Nieves, "Current-voltage characteristic of electrospray processes in microfluidics," Phys. Rev. Lett. 105, 154503 (2010).

${ }^{15}$ A. S. Utada, E. 1. Lorenceau, D. Link, P. Kaplan, H. Stone, and D. Weitz, "Monodisperse double emulsions generated from a microcapillary device," Science 308, 537-541 (2005).

${ }^{16}$ A. Barrero, J. M. Lopez-Herrera, A. Boucard, I. G. Loscertales, and M. Marquez, "Steady cone-jet electrosprays in liquid insulator baths," J. Colloid Interface Sci. 272, 104-108 (2004).

${ }^{17}$ The Reynolds numbers are usually very small, and thus the densities of the liquids play no role.

${ }^{18}$ A. S. Utada, A. Fernandez-Nieves, H. A. Stone, and D. A. Weitz, "Dripping to jetting transitions in coflowing liquid streams," Phys. Rev. Lett. 99, 094502 (2007).

${ }^{19}$ N. Vilanova, V. R. Gundabala, and A. Fernandez-Nieves, "Drop size control in electro-coflow," Appl. Phys. Lett. 99, 021910 (2011).

${ }^{20}$ A. J. Hijano, I. G. Loscertales, S. E. Ibanez, and F. J. Higuera, "Periodic emission of droplets from an oscillating electrified meniscus of a low-viscosity, highly conductive liquid," Phys. Rev. E 91, 013011 (2015).

${ }^{21}$ P. K. Notz and O. A. Basaran, "Dynamics of drop formation in an electric field," J. Colloid Interface Sci. 213, 218-237 (1999).

${ }^{22}$ J. Happel and H. Brenner, Low Reynolds Number Hydrodynamics (PrenticeHall, New Jersey, 1983), pp. 227-230.

${ }^{23}$ L. Rayleigh, "On the equilibrium of conducting masses charged with electricity," Philos. Mag. 14, 184-186 (1882).

${ }^{24}$ F. K. Wohlhuter and O. A. Basaran, "Shapes and stability of pendant and sessile dielectric droplets in an electric field," J. Fluid Mech. 235, 481-510 (1992).

${ }^{25}$ J. Guerrero, J. Rivero, V. R. Gundabala, M. Perez-Saborid, and A. FernandezNieves, "Whipping of electrified liquid jets," Proc. Natl. Acad. Sci. U. S. A. 111, 13763-13767 (2014).

${ }^{26}$ T. Cubaud, B. M. Jose, and S. Darvishi, "Folded micro-threads: Role of viscosity and interfacial tension," Phys. Fluids 23, 042002 (2011).

${ }^{27}$ G. Riboux, A. G. Marin, I. G. Loscertales, and A. Barrero, "Whipping instability characterization of an electrified visco-capillary jet," J. Fluid Mech. 671, 226 (2011).

${ }^{28}$ M. Hohman, M. Shin, G. Rutledge, and M. Brenner, "Electrospinning and electrically forced jets. I. Stability theory," Phys. Fluids 13, 2201-2220 (2001). 